\title{
3D MODELLING OF ARCHAEOLOGICAL SMALL FINDS BY A LOW-COST RANGE CAMERA: METHODOLOGY AND FIRST RESULTS
}

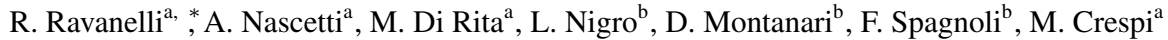 \\ ${ }^{a}$ Geodesy and Geomatics Division, DICEA - University of Rome "La Sapienza”, Rome, Italy \\ $<$ roberta.ravanelli, andrea.nascetti, martina.dirita, mattia.crespi $>@$ uniroma1.it \\ b Department of Oriental Studies, - University of Rome "La Sapienza" - Archaeological Expedition to Motya \\ $<$ lorenzo.nigro, daria.montanari, federica.spagnoli >@uniroma1.it
}

KEY WORDS: Close range photogrammetry, 3D modelling, Range Cameras, Structure Sensor, Small Finds

\begin{abstract}
:
The production of reliable documentation of small finds is a crucial process during archaeological excavations. Range cameras can be a valid alternative to traditional illustration methods: they are veritable 3D scanners able to easily collect the 3D geometry (shape and dimensions in metric units) of an object/scene practically in real-time.

This work investigates precisely the potentialities of a promising low-cost range camera, the Structure Sensor ${ }^{\mathrm{TM}}$ by Occipital, for rapid modelling archaeological objects. The accuracy assessment was thus performed by comparing the 3D model of a Cipriot-Phoenician globular jug captured by this device with the 3D model of the same object obtained through photogrammetry.

In general, the performed analysis shows that Structure Sensor is capable to acquire the 3D geometry of a small object with an accuracy comparable at millimeter level to that obtainable with the photogrammetric method, even though the finer details are not always correctly modelled. The texture reconstruction is instead less accurate. In the end, it can be concluded that the range camera used for this work, due to its low-cost and flexibility, is a suitable tool for the rapid documentation of archaeological small finds, especially when not expert users are involved.
\end{abstract}

\section{INTRODUCTION}

Range cameras are active imaging sensors, low-cost and easy-touse, able to natively measure the distances of several points at high frame rate $(30-60 \mathrm{~Hz})$. At every acquisition, they produce the depth map of the scene, an image in which each pixel contains its own distance from a specific reference, normally associated to the sensor itself (generally the image plane). Starting from this depth map, range cameras capture a dense point cloud, that is a collection of an elevate number of 3D coordinates into a local reference system, of the scanned environment.

Therefore, according to the definition given in (Boehler and Marbs, 2002), range cameras can be considered, to all intents and purposes, veritable 3D scanners. Furthermore, Simultaneous Localization And Mapping (SLAM) algorithms, such us KinectFusion ((Izadi et al., 2011), (Newcombe et al., 2011)), leverage the depth data and the high frame rate that range cameras offer, in order to fuse the depth maps captured from different view points as soon as they are acquired. In this way, the depth maps are merged into an overall 3D model easily and practically in real-time by continually estimating the pose of the moving sensor. In addition, such sensors are continually evolving and they will be soon integrated in consumer grade smart devices, enabling their use together with other sensors (see, for example, (Benedetti et al., 2016), (Ravanelli et al., 2016)).

Thanks to all these features, nowadays this technology is sufficiently ripe to play an important role for modelling archaeological objects. Indeed, range cameras can be easily used for documenting small finds (with an extent greater than $10 \mathrm{~cm}$ ), thus representing a valid alternative to the often time consuming traditional techniques, and preserving at the same time the mental energy of archaeologists for the study and interpretation of the artifacts discovered during excavations. Anyway, before using

${ }^{*}$ Corresponding author. systematically these devices on the field, it is essential to assess the metric quality of their 3D geometry reconstruction. This work investigates precisely the $3 \mathrm{D}$ modelling capabilities of a promising low-cost range camera, the Structure Sensor ${ }^{\mathrm{TM}}$ by Occipital (Figure 1).

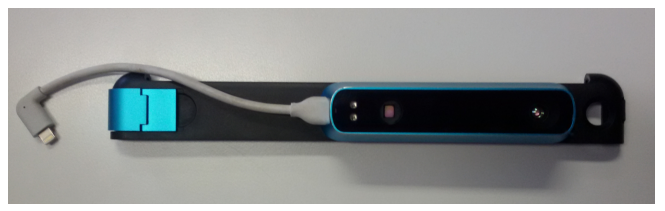

Figure 1: The Structure Sensor by Occipital.

Launched on Kickstarter in September 2013 and raising almost 1.3 millions of dollars in 45 days of campaign, the Structure Sensor has been conceived from the beginning to be the first range camera for mobile devices. Characterized by compact dimensions and an internal battery, it can be quickly and securely connected to an iOS device, thus making the range camera technology easily accessible to a wider and inexpert public. In particular, the Structure Sensor is a Structured Light range camera. It consists of an infrared laser projector and a frequency-matched infrared camera. The first emits a pattern of thousands of invisible infrared dots on the surface of the object/s to be modelled, whereas the latter records how the environment deforms the original pattern, thereby obtaining the 3D geometry (shape and dimensions in metric units) of the objects. Anyway, differently from the other range cameras available on the market, the Structure Sensor does not have its own colour camera and therefore the object texture is captured by the colour camera of the tablet/smartphone to which it is connected.

\section{3D MODEL COLLECTION AND ELABORATION}

In order to evaluate the quality of the 3D geometry reconstruction carried out by the Structure Sensor, the 3D model of a Cipriot- 
Phoenician globular jug (IX-VIII century B.C.) captured by this low-cost range camera was compared to the $3 \mathrm{D}$ model of the same object obtained with the photogrammetric technique. The jug, characterized by a rather shiny surface and the presence of several breaks (see Figure 2), is conserved at the Museum of the Near East, Egypt and Mediterranean of the University of Rome "La Sapienza" (Nigro, 2015) directed by Prof. L. Nigro.

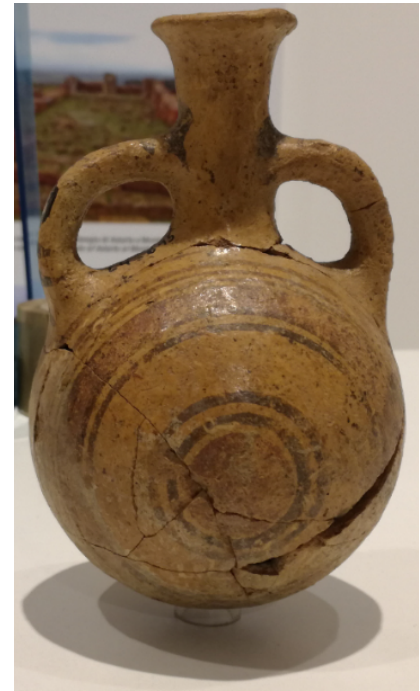

Figure 2: The Cipriot-Phoenician globular jug (IX-VIII century B.C.) used to test the 3D modelling capabilities of the Structure Sensor.

For the Structure Sensor, the Scanner app was selected among all the 3D scanning apps at the moment available for the device. Free and easy to use, it allows to capture in real time the 3D models of objects and people by simply walking around them with an iOS device connected to the Structure Sensor. The app is an integral part of the Structure SDK provided by Occipital: the source code is available in the form of sample examples and can be customized by the developers according to the specific requirements of the application.

In particular, the 3D model generation relies on the tracking, the process by which the sensor reliably estimates its own motion in relation to the object to be scanned, since multiple scans from different points of view are required to collect complete information about the whole surface of the object. Therefore, the user must slowly move around the target object, following a $360^{\circ}$ path and not forgetting to scan all the sides (including the top and the bottom). In most cases, the needed scanning time is about few minutes, depending on the object shape and complexity. Furthermore, it is possible to coordinate the movements of the sensor with the Augmented Reality live view on the screen of the iOS device and check the model quality immediately, during the very same scan. In this way the operator can improve the results instantly, by scanning again the problematic areas and filling the model holes. Finally, the operator should always check the dimensions of the scanning volume which can be usually restricted or enlarged in relation to the target object size. In order to obtain a most accurate 3D model, it is strongly recommended not to waste resolution and thus the scanning volume should be just a little bit wider than the object within it.

Anyway, before proceeding with the scan, the Structure Sensor was calibrated using the Calibrator app provided by Occipital and specifically designed for the Structure Sensor bracket accessory. In fact the object geometry and its color are captured from two different points of view and thus, in order to accurately overlap the 3D data with the corresponding texture, it is necessary to estimate the parameters of the geometric relationship between the Structure Sensor and the iOS device camera. The calibration is important also for a successful outcome of the object scan, since it can influence the correct functioning of the tracking. In general, indeed, the tracking can leverage both the geometry of the objects to be scanned and their colour data. The Structure Sensor calibration and the scanning of the jug were performed in less than 10 minutes.

As regards the photogrammetric method, it was carried out by processing 77 images with the Agisoft Photoscan software (Agisoft PhotoScan, 2017). The images were captured by the camera of a smartphone with a resolution of $4160 \times 3120$ pixels, a focal length of $3.79 \mathrm{~mm}$ and a CCD pixel dimension of $0.001168 \mathrm{~mm}$. During the image orientation process the camera parameters were refined considering both radial and tangential coefficient. Moreover, three scale bar ranging from 5 to $10 \mathrm{~cm}$ have been used in order to constrain the solution and to achieve a robust block alignment; the overall residual in terms of re-projection errors are close to 0.1 pixels.

Starting from the oriented block, the dense cloud and the 3D mesh were generated. In particular, two models were produced: one at very high and one at medium resolution in order to compare both of them with the 3D model obtained with the Structure Sensor. It is important to underline the differences in terms of processing time: less than 30 minutes for the medium resolution and 4-5 hours for the very high resolution model using a standard laptop (MacBook Pro Retina 2013).

The 3D model captured with the Structure Sensor and the two obtained with the photogrammetric technique are reported in Figure 5(a), Figure 5(b) and Figure 5(c) respectively.

As expected, the high resolution photogrammetric model is the most complete both for the 3D geometry reconstruction and for the texture rendering (for further details see Table 1).

Table 1: 3D models main features.

\begin{tabular}{lcccc}
\hline 3D model & points & faces & texture coords & normals \\
\hline Structure Sensor & 6405 & 9145 & 6405 & 6405 \\
AGI high & 620757 & 1239414 & 668985 & 620721 \\
AGI medium & 26754 & 53338 & 30245 & 26753 \\
\hline
\end{tabular}

Once the 3D models were generated, they were exported in the .OBJ format and their meshes were registered through the Iterative Closest Point (ICP) algorithm (Best and McKay, 1992) implemented in the CloudCompare (Girardeau-Montaut, 2017) software. In particular, the parameters of the roto-translation and the scale were estimated, but only the first ones were used to align the models, since the scale was not significant. In this way, it was possible to evaluate the $3 \mathrm{D}$ reconstruction accuracy in terms of signed distances (positive inside and negative outside) of the points of the Structure Sensor model from the mesh of each of the two photogrammetric models. The computed distances, the related histograms and the statistics are reported in Figure 3, Figure 4 and Table 2 .

Table 2: 3D distance models comparison (distance statistics).

\begin{tabular}{lcccccccc}
\hline & $\begin{array}{c}\text { mean } \\
{[\mathrm{m}]}\end{array}$ & $\begin{array}{c}\text { st. dev. } \\
{[\mathrm{m}]}\end{array}$ & $\begin{array}{c}\text { median } \\
{[\mathrm{m}]}\end{array}$ & $\begin{array}{c}\text { NMAD } \\
{[\mathrm{m}]}\end{array}$ & $\begin{array}{c}\text { LE68 } \\
{[\mathrm{m}]}\end{array}$ & $\begin{array}{c}\text { LE90 } \\
{[\mathrm{m}]}\end{array}$ & $\begin{array}{c}\min \\
{[\mathrm{m}]}\end{array}$ & $\begin{array}{c}\max \\
{[\mathrm{m}]}\end{array}$ \\
\hline SS vs AGI high & 0.000 & 0.001 & 0.000 & 0.001 & 0.001 & 0.002 & 0.009 & -0.011 \\
SS vs AGI medium & 0.000 & 0.001 & 0.000 & 0.001 & 0.001 & 0.002 & 0.003 & -0.008 \\
\hline
\end{tabular}

The distance mean and standard deviation are equal to $0 \mathrm{~mm}$ and $1 \mathrm{~mm}$ for both the two analyzed cases. Moreover, both of the histograms are bimodal and this behaviour is probably due, at least 


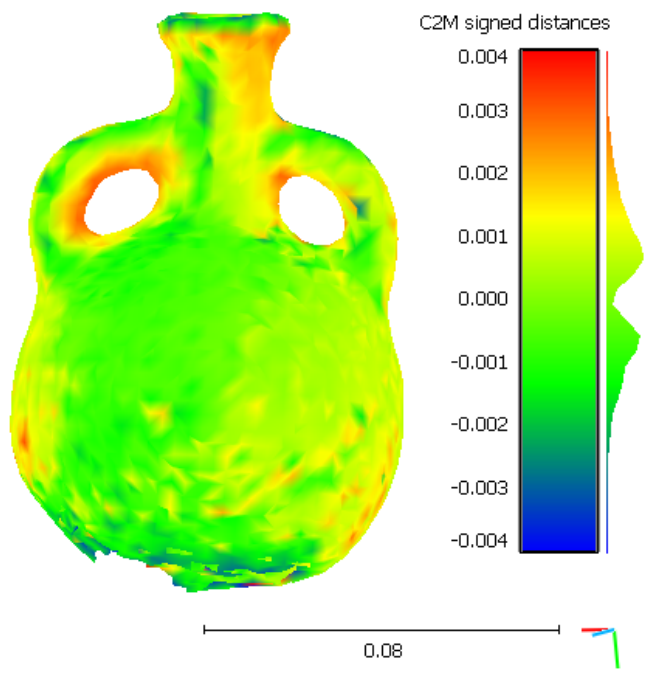

(a)

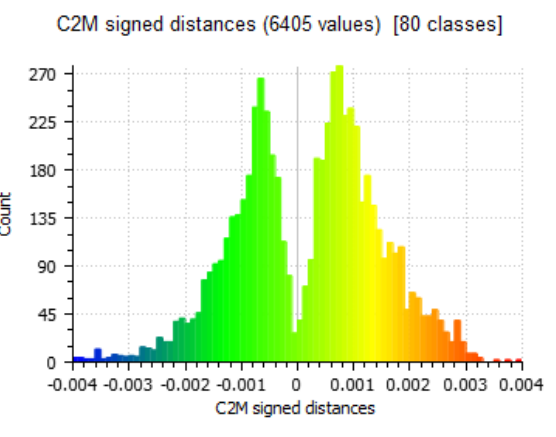

(b)

Figure 3: (a) Distances between the points of the Structure Sensor model and the mesh of the high resolution photogrammetric model; (b) related histogram.

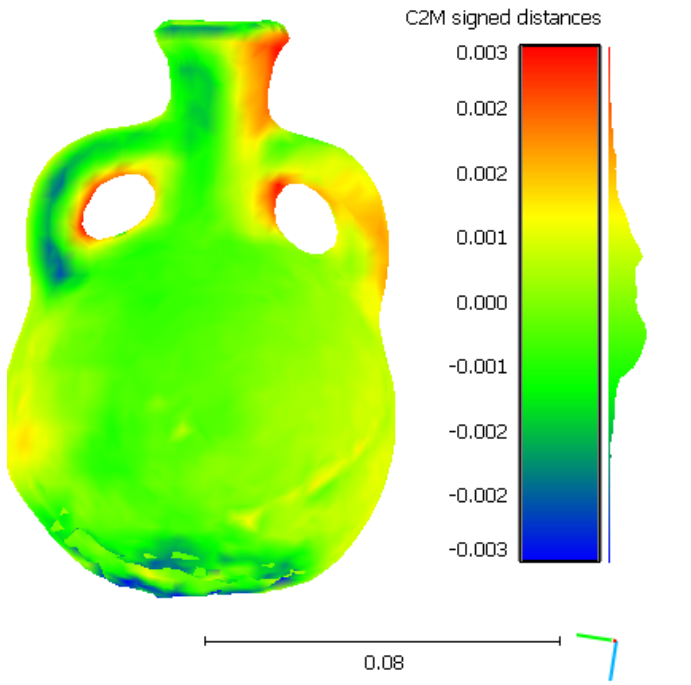

(a)

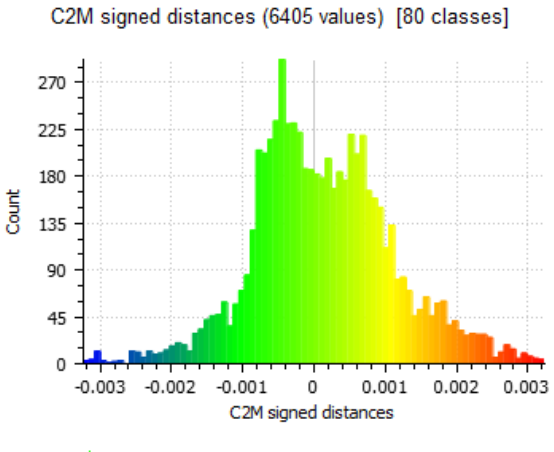

(b)

Figure 4: (a) Distances between the points of the Structure Sensor model and the mesh of the medium resolution photogrammetric model; (b) related histogram.

in part, to residual registration errors. Furthermore, from what is visible in Figure 3 and Figure 4, the most different areas are located in correspondence of the handles, the base and the cracks, while the remaining surfaces are generally quite similar. For what regards the base, the higher distances values are probably caused by the different holes in correspondence of the pedestal, where it was not possible to completely capture the object neither with the Structure Sensor nor with the smartphone camera. Regarding the cracks, the Structure Sensor was not able to model them: they were too small for the Scanner app, which, like almost all the 3D scanning applications, implements a strategy specifically conceived to close the holes.

The distances computed with the Cloud Compare approach provide general indications about the quality of the 3D geometry reconstruction carried out by the Structure Sensor. Anyway, in order to locally characterize the accuracy of this low-cost range camera, two quantities of archaeological interest were measured on both the Structure Sensor model and the photogrammetric ones. In particular, two sections were cut in correspondence of the entrance and the maximum width of the jug, in order to measure the related diameters. The results are reported in Table 3 and they generally confirm the good performances of the Structure Sensor, once again at millimeter level.

Table 3: Diameter measurements.

\begin{tabular}{lcccc}
\hline & \multicolumn{2}{c}{ Max width section } & \multicolumn{2}{c}{ Entrance section } \\
\hline 3D model & $\mathrm{D}_{1}$ & $\mathrm{D}_{2}$ & $\mathrm{D}_{1}$ & $\mathrm{D}_{2}$ \\
& {$[\mathrm{~m}]$} & {$[\mathrm{m}]$} & {$[\mathrm{m}]$} & {$[\mathrm{m}]$} \\
\hline SS high & 0.076 & 0.088 & 0.030 & 0.030 \\
AGI high & 0.078 & 0.086 & 0.030 & 0.030 \\
SS medium & 0.075 & 0.088 & 0.030 & 0.031 \\
AGI medium & 0.077 & 0.087 & 0.031 & 0.029 \\
\hline
\end{tabular}

Finally, the texture reconstruction is instead less accurate. In- 


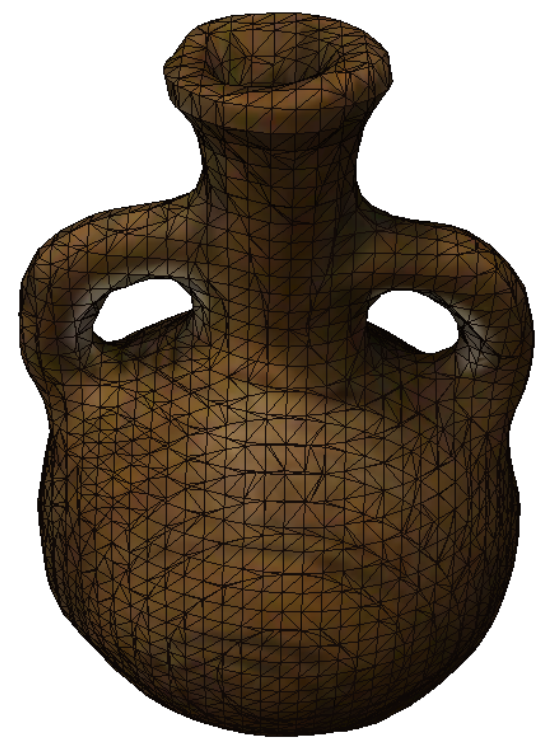

(a)

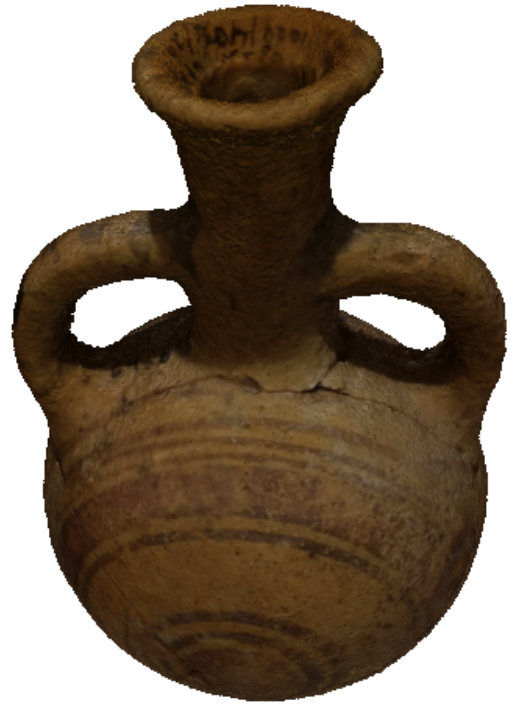

(b)

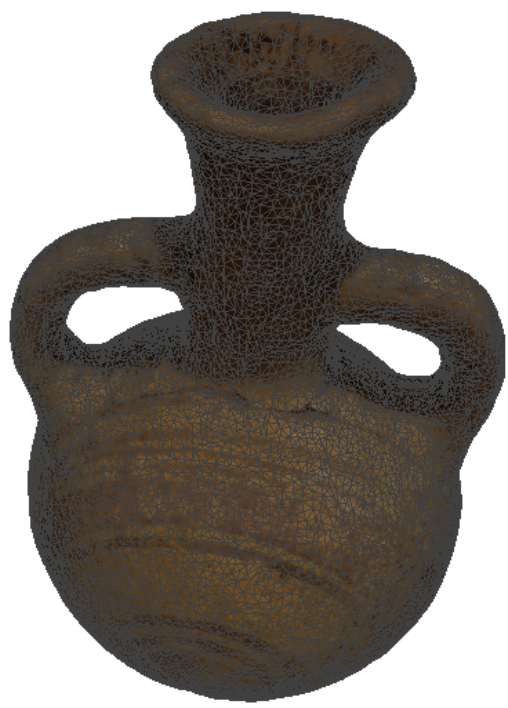

(c)

Figure 5: The generated 3D models: (a) solid wireframe visualization of the Structure Sensor model; (b) solid visualization of the high resolution photogrammetric model; (c) solid wireframe visualization of the medium resolution photogrammetric model.

deed, the colouring approach used by the Scanner app of Occipital seems to smooth the texture details; occasionally the colour is not perfectly aligned to the $3 \mathrm{D}$ geometry in some areas of the model, in particular for those captured at end of the scanning process (at the end of the $360^{\circ}$ path). This behaviour can be explained with a not perfect outcome of the calibration, residual tracking errors and/or motion blur effects occurred when capturing key frames.

\section{CONCLUSION AND FUTURE WORKS}

Our preliminary results are encouraging. The Structure Sensor is capable to acquire the $3 \mathrm{D}$ geometry of a small object in quasi real-time (few minutes) with an accuracy comparable at millimeter level to that obtainable with the more complex (for not expert users) and lengthy (from 30 minutes to $4-5$ hours) photogrammetric method.

However, the colors are not fully rendered and the finer details such as the cracks are not always correctly modelled. Anyway, the texture issue is due to the coloring approach implemented in the software used and it can be surely solved, as it already happens with other not free and proprietary 3D scanning applications (for instance, see Skanect or itSeez3D).

In conclusion, the Stucture Sensor is a suitable tool for the rapid documentation of small archaeological finds, especially when not expert users are involved, due to its low-cost and flexibility.

Finally, as a future work, it would be worthy testing the Structure Sensor with objects of different size and with other 3D scanning applications.
Best, P. J. and McKay, N. D., 1992. A Method for Registration of 3-D Shapes. IEEE Transactions on pattern analysis and machine intelligence 14(2), pp. 239-256.

Boehler, W. and Marbs, A., 2002. 3D scanning instruments. Proceedings of the CIPA WG 6, pp. 9-18.

Girardeau-Montaut, D., 2017. Cloud Compare - 3D Point Cloud and Mesh Processing Software - Version 2.8.1. Open Source Project.

Izadi, S., Kim, D., Hilliges, O., Molyneaux, D., Newcombe, R., Kohli, P., Shotton, J., Hodges, S., Freeman, D., Davison, A. and Fitzgibbon, A., 2011. KinectFusion: Real-time 3D Reconstruction and Interaction Using a Moving Depth Camera. Proceedings of the 24th annual ACM symposium on User interface software and technology.

Newcombe, R., Izadi, S., Hilliges, O., Molyneaux, D., Kim, D., Davison, A., Kohli, P., Shotton, J., Hodges, S. and Fitzgibbon, A., 2011. KinectFusion: Real-Time Dense Surface Mapping and Tracking. Proceedings of the 10th IEEE International Symposium on Mixed and Augmented Reality (ISMAR).

Nigro, L., 2015. Il nuovo allestimento del museo del vicino oriente, egitto e mediterraneo della Sapienza. Vicino Oriente XIX pp. $309-340$

Ravanelli, R., Nascetti, A. and Crespi, M., 2016. Kinect V2 And RGB Stereo Cameras Integration For Depth Map Enhancement. ISPRS - International Archives of the Photogrammetry, Remote Sensing and Spatial Information Sciences XLI-B5, pp. 699-702.

\section{REFERENCES}

Agisoft PhotoScan, 2017. http://www.agisoft.com/.

Benedetti, E., Ravanelli, R., Moroni, M., Nascetti, A. and Crespi, M., 2016. Exploiting Performance of Different Low-Cost Sensors for Small Amplitude Oscillatory Motion Monitoring: Preliminary Comparisons in View of Possible Integration. Journal of Sensors 2016, pp. 10. 